\title{
Numerical Optimal Control of Wave Energy Converters
}

\author{
Giorgio Bacelli and John V. Ringwood, Senior Member, IEEE
}

\begin{abstract}
Energy maximizing control for wave energy converters (WECs) is a nonstandard optimal control problem. While the constrained optimal control problem for WECs has been addressed by model-predictive control strategies, such strategies need to employ cost function modifications due to convexity problems and the algorithms are computationally complex, making real-time implementation difficult. The recently developed family of direct transcription methods offer a promising alternative, since they are computationally efficient and a convex problem results. Moreover, constraints on both the device displacement and velocity, and power take off force, are easily incorporated. Both single-body and multibody device models can be used, as well as arrays of single-body or multibody devices.
\end{abstract}

Index Terms-Control systems, direct transcription, wave energy.

\section{INTRODUCTION}

$\mathbf{N}$ OT unlike the energy maximization problem for wind turbines, the optimal control problem for wave energy converters (WECs) involves the maximization of a cost functional of the form

$$
J=-\int_{0}^{T} \dot{\boldsymbol{\eta}}(t)^{\top} \boldsymbol{f}_{\mathrm{pto}}(t) d t
$$

where $\dot{\boldsymbol{\eta}}(t)$ is the device velocity, $\boldsymbol{f}_{\text {pto }}(t)$ is the power takeoff (PTO) force, and $J$ represents the useful energy converted by the PTO. However, (1) is a departure from the standard optimal (regulator) control problem, which is normally quadratic in the arguments of the integral, and therefore, represents a convex optimization problem.

Early work on the optimal constrained control of WECs in irregular waves focussed on the application of Pontryagin's maximum principle and the numerical solution, using Lagrange multipliers, to the resulting two-point boundary value problem [1]. The main difficulty with such indirect methods (which attempt to solve the necessary conditions for optimality) is that the set of necessary conditions for optimality, which must be

Manuscript received May 12, 2014; revised July 30, 2014 and November 10, 2014; accepted November 12, 2014. Date of publication December 18, 2014; date of current version March 18, 2015. This work was supported in part by Enterprise Ireland under Grant EI/TD/2009/0331. This work was completed while G. Bacelli was affiliated with the Centre for Ocean Energy Research at Maynooth University. Paper no. TSTE-00220-2014.

G. Bacelli is with the Water Power Technologies Department, Sandia National Laboratories, Albuquerque, NM 87123 USA (e-mail: gbacelli@ sandia.gov).

J. V. Ringwood is with the Centre for Ocean Energy Research, Maynooth University, Maynooth, Ireland (e-mail: john.ringwood@eeng.nuim.ie).

Digital Object Identifier 10.1109/TSTE.2014.2371536 derived analytically, are problem-specific and the region of convergence for the optimal solution is small, requiring a good initial guess.

More recently, the popular model predictive control (MPC) philosophy has been adapted for use by the wave energy community, e.g., [2]-[4]. However, attempts to adopt discrete-time versions of performance functional of (1) have attracted various modifications, including the specification of a time offset between $\dot{\boldsymbol{\eta}}(t)$ and $\boldsymbol{f}_{\text {pto }}(t)$ (namely, $\dot{\boldsymbol{\eta}}(k+1) \boldsymbol{f}_{\text {pto }}(k)$, where $k$ is the discrete time index [3], or the addition of extra terms to make the cost functional quadratic [4].

This paper describes the application of a direct transcription method to the optimal control of WECs and develops a generalized discretization framework within which the WEC control problem can be solved. The transcription method used for the discretization of the control problem employs the mean-weighted residuals as described in [5]. The first reported application of a direct transcription method to the wave energy control problem was in [6], which parameterizes device motion and PTO force using Fourier basis functions (and employs amplitude constraints only), while [7] uses a pseudospectral method to approximate motion and PTO force using polynomials, requiring the addition of a further constraint to ensure periodicity.

This paper proceeds as follows. The discretization is carried out for a generic configuration of WECs and generic sets of basis and test functions; detailed derivations of the quadratic program resulting from the direct transcription are also included here. As an illustrative example, Section III describes the application of the Galerkin method to a single body WEC, where the basis functions are truncated Fourier series. Derivations of the matrices composing the quadratic program, and a discussion about some of their properties are also illustrated (e.g., convexity).

Simulation results are presented in Section III-B for both regular and irregular incident waves, including restrictions on both PTO force and oscillation amplitude. Section IV provides a general discussion on the computational aspects associated with the algorithm, with Section IV-A, in particular, providing a simplification of the convolution integral associated with the radiation force, when the velocity is approximated with a generic expansion. It is shown that, because of linearity, the computations involving the numerical integration of the convolution integral can be carried out offline, thus significantly reducing the computational load when solving the nonlinear programming (NLP) problem. 


\section{General Case: Mean-Weighted Residual FORMULATION OF A SYSTEM OF WECS}

The objective of this section is to present the steps for the direct transcription of the optimal control problem of a generic system WECs, the model of which is described by Cummins' equation [8]

$$
\begin{aligned}
& \boldsymbol{M} \ddot{\boldsymbol{\eta}}(t)+\boldsymbol{B} \dot{\boldsymbol{\eta}}(t)+\int_{0}^{t} \boldsymbol{K}(t-\tau) \dot{\boldsymbol{\eta}}(\tau) d \tau+\boldsymbol{S} \boldsymbol{\eta}(t) \\
& \quad=\tilde{\boldsymbol{f}}_{p}(t)+\boldsymbol{f}_{e}(t)
\end{aligned}
$$

where $\boldsymbol{\eta}(t) \in \mathbb{R}^{n}$ is the position vector of the WEC and $n$ is the number of degrees of freedom of the system. The matrix $M \in \mathbb{R}^{n \times n}$ is the sum of the generalized mass matrix and the added mass at infinite frequency; $\boldsymbol{B}$ and $\boldsymbol{S}$ are positive constant $n \times n$ diagonal matrices describing the linear damping and the stiffness, respectively. The elements of the $n \times n$ matrix of radiation impulse responses $\boldsymbol{K}(t)$ are continuous functions in $[0, \alpha)$ and zero for $t<0$, where $\alpha \leq \infty$.

The vector of excitation forces $\boldsymbol{f}_{e}(t):[0, t] \rightarrow \mathbb{R}^{n}$ is assumed to be continuous, and the vector of the PTO forces is considered to be $\tilde{\boldsymbol{f}}_{p}(t)=\boldsymbol{F}_{P} \boldsymbol{f}_{\text {pto }}$, where $\boldsymbol{F}_{P}$ is an $n \times m$ constant matrix. The role of the matrix $\boldsymbol{F}_{P}$ is to allow a general combinations of PTO components, particularly where the number of PTO forces $(m)$ are different from the number of modes of oscillation of the system $(n)$. In general, there are less PTO forces than modes of oscillations $(m \leq n)$. A more detailed explanation regarding the matrix $\mathbf{F}_{P}$ will be provided in Section II-A. The matrix $\boldsymbol{F}_{P}$ also takes part in the definition of the total absorbed energy over the time interval $[0, T]$, which is defined as the sum of the mechanical work done by all the PTO forces

$$
J=-\int_{0}^{T} \dot{\boldsymbol{\eta}}(t)^{\top} \boldsymbol{F}_{P} \boldsymbol{f}_{\mathrm{pto}}(t) d t .
$$

In practice, $\boldsymbol{F}_{P}$ specifies how the PTO forces and the velocities are combined to produce the usable power.

The optimal control problem is to find the PTO force vector $f_{\text {pto }}$ that maximizes the total absorbed energy $J$, subject to the equation of motion (2) and the additional control and motion path constraints described as

$$
\begin{aligned}
\boldsymbol{h}\left(\boldsymbol{\eta}, \dot{\boldsymbol{\eta}}, \boldsymbol{f}_{\mathrm{pto}}, t\right) & =0 \\
\boldsymbol{g}\left(\boldsymbol{\eta}, \dot{\boldsymbol{\eta}}, \boldsymbol{f}_{\mathrm{pto}}, t\right) & \leq 0 .
\end{aligned}
$$

Before proceeding with the discretization of the optimal control problem, the equation of motion (2) has to be rewritten as a system of first-order integro-differential equations. This is achieved, as usual, by introducing an additional state variable describing the velocity $(\boldsymbol{v})$, and the resulting system dynamic is

$$
\begin{gathered}
\dot{\boldsymbol{\eta}}=\boldsymbol{v} \\
\boldsymbol{M} \dot{\boldsymbol{v}}=-\boldsymbol{B} \boldsymbol{v}-\int_{-\infty}^{t} \boldsymbol{K}(t-\tau) \boldsymbol{v}(\tau) d \tau-\boldsymbol{S} \boldsymbol{\eta}+\boldsymbol{F}_{P} \boldsymbol{f}_{\mathrm{pto}}+\boldsymbol{f}_{e}
\end{gathered}
$$

The discretization of the control problem is performed by approximating the position $(\boldsymbol{\eta})$ and the velocity $(\boldsymbol{v})$ vectors with a linear combination of the basis functions $\phi_{k}(t)$, and the PTO force $\left(f_{\text {pto }}\right)$ vector with a linear combination of the functions $\phi_{k}^{P}(t)$; the $i$ th components of these vectors are

$$
\begin{gathered}
\eta_{i}(t) \approx \eta_{i}^{N}(t)=\sum_{k=1}^{N} x_{i k}^{\eta} \phi_{k}(t)=\Phi(t) \hat{\boldsymbol{X}}_{i}^{\eta}, \\
i=1, \ldots, n \\
v_{i}(t) \approx v_{i}^{N}(t)=\sum_{k=1}^{N} x_{i k}^{v} \phi_{k}(t)=\Phi(t) \hat{\boldsymbol{X}}_{i}^{v}, \\
i=1, \ldots, n \\
f_{\mathrm{pto}_{i}}(t) \approx f_{\mathrm{pto}_{i}}^{N^{P}}(t)=\sum_{k=1}^{N^{P}} u_{i k} \phi_{k}^{P}(t)=\Phi^{P}(t) \hat{\boldsymbol{u}}_{i}, \\
i=1, \ldots, m
\end{gathered}
$$

where $N$ and $N^{P}$ are the orders of the expansions for the states (position and velocity) and the control input (PTO force), respectively

$$
\begin{gathered}
\hat{\boldsymbol{X}}_{i}^{\eta}=\left[\hat{x}_{i 1}^{\eta}, \hat{x}_{i 2}^{\eta}, \ldots, \hat{x}_{i N}^{\eta}\right]^{\top}, \quad \hat{\boldsymbol{X}}_{i}^{v}=\left[\hat{x}_{i 1}^{v}, \hat{x}_{i 2}^{v}, \ldots, \hat{x}_{i N}^{v}\right]^{\top} \\
\hat{\boldsymbol{u}}_{i}=\left[\hat{u}_{i 1}, \hat{u}_{i 2}, \ldots, \hat{u}_{i N_{P}}\right]^{\top}
\end{gathered}
$$

and

$$
\begin{aligned}
\Phi(t) & =\left[\phi_{1}(t), \phi_{2}(t), \ldots, \phi_{N}(t)\right] \\
\Phi^{P}(t) & =\left[\phi_{1}^{P}(t), \phi_{2}^{P}(t), \ldots, \phi_{N_{P}}^{P}(t)\right] .
\end{aligned}
$$

Using the approximated velocity and PTO force, from (9) and (10), respectively, the total absorbed energy $J^{N}$ is

$$
J^{N}=-\int_{0}^{T} \Phi(t) \boldsymbol{X}^{v} \boldsymbol{F}_{p} \boldsymbol{U}^{\top} \Phi^{P^{\top}}(t) d t=\sum_{i=1}^{N^{P}} \sum_{j=1}^{N} w_{i j} \Gamma_{i j}
$$

where $w_{i j}$ are the elements of the matrix $\boldsymbol{W}=\boldsymbol{X}^{v} \boldsymbol{F}_{p} \boldsymbol{U}^{\top}$, which depends on the coefficients of the velocity $\left(\boldsymbol{X}^{v}\right)$ and the PTO force $(\boldsymbol{U})$, while $\Gamma_{i j}$ are the elements of the constant matrix $\Gamma$ which depends on the bases $\Phi$ and $\Phi^{P}$ as

$$
\Gamma=\int_{0}^{T} \Phi^{\top}(t) \Phi^{P}(t) d t
$$

The matrices $\boldsymbol{X}^{\eta}, \boldsymbol{X}^{v}$, and $\boldsymbol{U}$ are defined as

$$
\begin{gathered}
\boldsymbol{X}^{\eta}=\left[\hat{\boldsymbol{x}}_{1}^{\eta}, \ldots, \hat{\boldsymbol{x}}_{N}^{\eta}\right], \quad \boldsymbol{X}^{v}=\left[\hat{\boldsymbol{x}}_{1}^{v}, \ldots, \hat{\boldsymbol{x}}_{N}^{v}\right] \\
\boldsymbol{U}=\left[\hat{\boldsymbol{u}}_{1}, \ldots, \hat{\boldsymbol{u}}_{N_{P}}\right] .
\end{gathered}
$$

The derivatives of the approximated state variables (positions and velocities) are

$$
\begin{aligned}
& \dot{\eta}_{i}^{N}(t)=\sum_{k=1}^{N} x_{i k}^{\eta} \dot{\phi}_{k}(t)=\dot{\Phi}(t) \hat{\boldsymbol{x}}_{i}^{\eta}, \quad i=1, \ldots, n \\
& \dot{v}_{i}^{N}(t)=\sum_{k=1}^{N} x_{i k}^{v} \dot{\phi}_{k}(t)=\dot{\Phi}(t) \hat{\boldsymbol{x}}_{i}^{v}, \quad i=1, \ldots, n
\end{aligned}
$$

since the vectors $\hat{\boldsymbol{x}}_{i}^{\eta}$ and $\hat{\boldsymbol{x}}_{i}^{v}$ are independent of time. Substituting the approximated states (8) and (9), their 
derivatives (13), (14) and the PTO forces (10) into the dynamic equations (6) yields the residual form

$$
\begin{gathered}
r_{i}^{\eta}=\dot{\eta}_{i}^{N}-v_{i}^{N} \\
r_{i}^{v}=\sum_{j=1}^{n} m_{i j} \dot{v}_{j}^{N}+B_{i i} v_{i}^{N}+\sum_{j=1}^{n} \int_{0}^{t} K_{i j}(t-\tau) v_{j}^{N}(\tau) d \tau \\
+S_{i i} \eta_{i}^{N}(t)+\sum_{j=1}^{m} f_{p_{i j}} f_{\mathrm{pto}_{j}}^{N^{P}}+f_{e_{i}}
\end{gathered}
$$

where $m_{i j}$ are the elements of the matrix $M ; B_{i i}$ and $S_{i i}$ are the diagonal elements of the diagonal matrices $B$ and $S$, respectively; $f_{p_{i j}}$ are the elements of $\boldsymbol{F}_{P}$ and $K_{i j}$ are the elements of $\boldsymbol{K}$.

In applying the mean-weighted residual method, for any given values of the PTO force described by the coefficients $\boldsymbol{U}$, the coefficients of the velocity and position satisfying the equation of motion are calculated by solving the linear system of equations

$$
\begin{gathered}
\left\langle r_{i}^{\eta}, \psi_{j}\right\rangle=0 \\
\left\langle r_{i}^{v}, \psi_{j}\right\rangle=0, \quad \text { for } i=1, \ldots, n \text { and } j=1, \ldots, N
\end{gathered}
$$

where $\psi_{j}$ are linearly independent test functions and \langle\rangle denotes the inner product. In particular, two specific choices for the test functions lead to two well-known methods [5], which are the Galerkin or spectral method and the collocation or Pseudospectral method [9]. The Galerkin method is discussed in detail in Section III, whereas the pseudospectral method uses translated Dirac delta test functions, illustrated in [10], [11]. The system of equations (17) and (18) is linear because the dynamic equation is linear, and they form a system of $2 n N$ equations in $2 n N$ variables $\left(\boldsymbol{X}^{\eta}, \boldsymbol{X}^{v}\right)$.

The result of the discretization is the finite dimensional nonlinear program described by the quadratic cost function $J^{N}$ in (11), the linear equality constraints due to the dynamic equations in (17) and (18), and by the additional equality and inequality path and control constraints in (4) and (5) which are now functions of the vectors $\boldsymbol{X}^{\eta}, \boldsymbol{X}^{v}, \boldsymbol{U}$ and time, as

$$
\begin{aligned}
& \boldsymbol{h}\left(\boldsymbol{X}^{\eta}, \boldsymbol{X}^{v}, \boldsymbol{U}, t\right)=0 \\
& \boldsymbol{g}\left(\boldsymbol{X}^{\eta}, \boldsymbol{X}^{v}, \boldsymbol{U}, t\right) \leq 0 .
\end{aligned}
$$

\section{A. Definition of the PTO Configuration Matrix}

The role of the matrix $\boldsymbol{F}_{P}$ in (3) and (7) is to combine the PTO forces and the velocities of the oscillating modes for which energy is absorbed, and this section illustrates how to build the matrix for some common configurations of WECs.

In the case of a single-body device referenced to the seabed, such as the heaving buoy in Fig. 1, the system has one degree of freedom and there is only one velocity and one PTO force. The absorbed energy is

$$
J=\int_{0}^{T} \dot{z}(t) f_{\mathrm{pto}}(t) d t
$$

with $\boldsymbol{F}_{P}=1$. A self-reacting WEC composed of two heaving bodies, which are restricted to oscillate in heave only (Fig. 2),

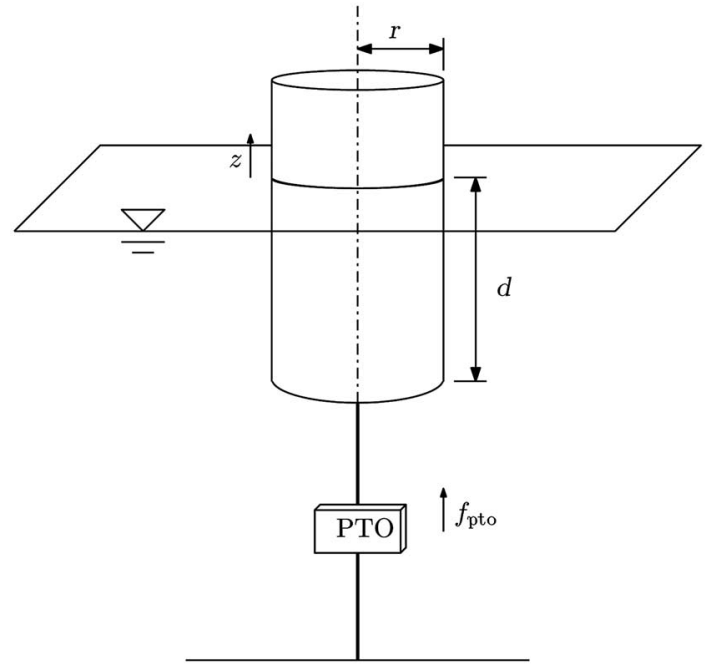

Fig. 1. Single-body heaving WEC.

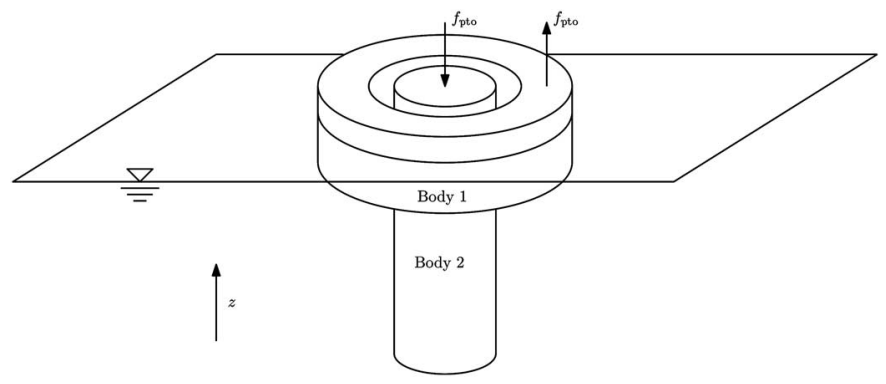

Fig. 2. Self-reacting and two-body heaving WEC.

has two degrees of freedom. In this two-body configuration, there is only one PTO force, and it acts on each of the bodies with the same magnitude but with opposite direction. If the system coordinates are the absolute vertical positions of the two bodies, then the absorbed energy is

$$
\begin{aligned}
J & =\int_{0}^{T}\left(\dot{z}^{B}(t)-\dot{z}^{A}(t)\right) f_{\text {pto }}(t) d t \\
& =\int_{0}^{T}\left[\dot{z}^{B}(t) \dot{z}^{A}(t)\right] \boldsymbol{F}_{P} f_{\text {pto }}(t) d t
\end{aligned}
$$

where $\dot{z}^{A}(t)$ and $\dot{z}^{B}(t)$ are the absolute vertical velocities of bodies $A$ and $B$, respectively; thus

$$
\boldsymbol{F}_{P}=\left[\begin{array}{c}
-1 \\
1
\end{array}\right]
$$

Additional illustrative examples, showing the procedure for the construction of the PTO configuration matrix for several WECs and arrays of WECs, are presented in [10].

\section{Fourier-GALERKIN DiRECT TRANSCRIPTION}

As an illustrative example, a single degree of freedom system describing the point absorber WEC depicted in Fig. 1 is considered [12], the motion of which is restricted to heave only; that is, the general WEC position vector $\eta$ is now the (scalar) heave 
position $z$. In this example, the Galerkin method is used in conjunction with trigonometric polynomials as approximating functions because the trigonometric polynomials, or truncated Fourier series, seem a natural choice for the oscillating system. For such a WEC system, the time-domain model of the WEC in (2) reduces to the scalar equation

$$
\begin{aligned}
M & \ddot{z}(t)+B \dot{z}(t)+\int_{-\infty}^{t} K(t-\tau) \dot{z}(\tau) d \tau+S z(t) \\
& =f_{e}(t)+f_{\text {pto }}(t)
\end{aligned}
$$

with $M=m_{b}+m_{\infty}$ and where $m_{b}$ is the mass of the oscillating body and $m_{\infty}$ is the added mass at infinite frequency for the heave mode. Since the PTO force is applied between the body and a fixed reference, the PTO configuration matrix is $\boldsymbol{F}_{P}=1$, and the absorbed energy is now defined as

$$
J=-\int_{0}^{T} \dot{z}(t) f_{\text {pto }}(t) d t .
$$

For convenience of notation, ${ }^{1}$ the state variables are renamed as $z=x_{1}$ and $\dot{z}=x_{2}$, and the PTO force, which is the control input, as $f_{\text {pto }}=u$; the resulting dynamic model is

$$
\begin{gathered}
\dot{x}_{1}=x_{2} \\
M \dot{x}_{2}=-B x_{2}-\int_{-\infty}^{t} K(t-\tau) x_{2}(\tau) d \tau-S x_{1}+u+f_{e} .
\end{gathered}
$$

The heave positions and velocities in (8) and (9), respectively, and the PTO force in (10), are approximated by zeromean truncated Fourier series with $N$ terms with $N=N^{P}$ for this case, i.e.,

$$
\begin{aligned}
x_{i}(t) & \approx \sum_{k=1}^{N / 2} x_{i k}^{c} \cos \left(k \omega_{0} t\right)+x_{i k}^{s} \sin \left(k \omega_{0} t\right)=\Phi(t) \hat{\boldsymbol{x}}_{i} \\
u(t) & \approx \sum_{k=1}^{N / 2} u_{k}^{c} \cos \left(k \omega_{0} t\right)+u_{k}^{s} \sin \left(k \omega_{0} t\right)=\Phi(t) \hat{\boldsymbol{u}}
\end{aligned}
$$

with $i=1,2$ and where

$$
\begin{aligned}
\hat{\boldsymbol{x}}_{i} & =\left[x_{i 1}^{c}, x_{i 1}^{s}, \ldots, x_{i \frac{N}{2}}^{c}+x_{i \frac{N}{2}}^{s}\right]^{\top} \\
\hat{\boldsymbol{u}} & =\left[u_{1}^{c}, u_{1}^{s}, \ldots, u_{\frac{N}{2}}^{c}+u_{\frac{N}{2}}^{s}\right]^{\top}
\end{aligned}
$$

and

$\Phi(t)=\left[\cos \left(\omega_{0} t\right), \sin \left(\omega_{0} t\right), \ldots, \cos \left(\frac{N}{2} \omega_{0} t\right), \sin \left(\frac{N}{2} \omega_{0} t\right)\right]$

where the fundamental frequency is $\omega_{0}=2 \pi / T$. The constant terms of the bases $(k=0)$ have been discarded because the exciting force is assumed to be zero-mean; therefore, the resulting (optimal) oscillation is also expected to be zero-mean.

By substituting the approximations in (24) and (25) into the expression for the total absorbed energy in (21), and by using (11), the approximated absorbed energy $J^{N}$ is

$$
J^{N}=-\int_{0}^{T} \hat{u}^{\top} \Phi^{\top}(t) \Phi(t) \hat{\boldsymbol{x}}_{2} d t=-\frac{T}{2} \hat{\boldsymbol{u}}^{\top} \hat{\boldsymbol{x}}_{2} .
$$

${ }^{1}$ in particular, for consistency with the notation used in the control theory.
In fact, the matrix $\Gamma$ in (12) is

$$
\Gamma=\int_{0}^{T} \Phi^{\top}(t) \Phi(t) d t=\frac{T}{2} I_{N}
$$

where $I_{N}$ is the identity matrix of size $N$, because the basis is orthogonal, i.e.,

$$
\left\langle\phi_{i}, \phi_{j}\right\rangle=\int_{0}^{T} \phi_{i}(t) \phi_{j}(t) d t=\frac{T}{2} \delta_{i j}
$$

where $\delta_{i j}$ is the Kronecker delta.

The next step of the discretization process is the construction of the linear system resulting from the minimization of the residual $r^{N}$ in (18) by applying the Galerkin method, as described in [5]. When using the Fourier series to approximate the states, the differentiation of the approximated states can be conveniently written as

$$
\dot{x}_{i}^{N}=\dot{\Phi}(t) \hat{\boldsymbol{x}}_{i}=\Phi(t) \boldsymbol{D}_{\phi} \hat{\boldsymbol{x}}_{i}
$$

where the differentiation matrix $D_{\phi} \in \mathbb{R}^{N \times N}$ is block diagonal. Each block $D_{\phi}^{k}$, for $k=1, \ldots, N / 2$ is

$$
\boldsymbol{D}_{\phi}^{k}=\left[\begin{array}{cc}
0 & k \omega_{0} \\
-k \omega_{0} & 0
\end{array}\right] .
$$

In fact, the derivative of a zero-mean Fourier series is still a Fourier series where the terms are reordered and scaled, since

$$
\frac{d}{d t} \sin (\omega t)=\omega \cos (\omega t) ; \quad \frac{d}{d t} \cos (\omega t)=-\omega \sin (\omega t) .
$$

Consequently, the approximated state equations in (22) and (23) become

$$
\begin{gathered}
\Phi(t) \boldsymbol{D}_{\phi} \hat{\boldsymbol{x}}_{1}=\Phi(t) \hat{\boldsymbol{x}}_{2} \\
M \Phi(t) \boldsymbol{D}_{\phi} \hat{\boldsymbol{x}}_{2}=-B \Phi(t) \hat{\boldsymbol{x}}_{2}-\int_{-\infty}^{t} K(t-\tau) \Phi(t) \hat{\boldsymbol{x}}_{2}(\tau) d \tau \\
-S \Phi(t) \hat{\boldsymbol{x}}_{1}+\Phi(t) \hat{\boldsymbol{u}}+f_{e} .
\end{gathered}
$$

The Galerkin method [5] consists of writing the dynamic equations in residual form, and then minimizing the residual by imposing its orthogonality to all the element of the basis. However, with regard to the first-state equation (28), it is possible to note immediately that

$$
\Phi(t) \boldsymbol{D}_{\phi} \hat{\boldsymbol{x}}_{1}-\Phi(t) \hat{\boldsymbol{x}}_{2}=0 \Leftrightarrow \boldsymbol{D}_{\phi} \hat{\boldsymbol{x}}_{1}-\hat{\boldsymbol{x}}_{2}=0
$$

since two Fourier series are equal if and only if all the corresponding coefficients are equal. The residual form of the second dynamic equation (29) is

$$
\begin{aligned}
r_{2}^{N}= & M \Phi(t) \boldsymbol{D}_{\phi} \hat{\boldsymbol{x}}_{2}+B \Phi(t) \hat{\boldsymbol{x}}_{2}+\int_{-\infty}^{t} K(t-\tau) \Phi(t) \hat{\boldsymbol{x}}_{2} d \tau \\
& +S \Phi(t) \hat{\boldsymbol{x}}_{1}-\Phi(t) \hat{\boldsymbol{u}}-f_{e}
\end{aligned}
$$

and is minimized by solving

$$
\left\langle r_{2}^{N}, \phi_{i}\right\rangle=0 \quad i=1, \ldots, N
$$


which can be written, since the inner product is commutative, in a more concise form as

$$
\left\langle\Phi^{\top}, r_{2}^{N}\right\rangle=\mathbf{0}
$$

where $\mathbf{0} \in \mathbb{R}^{N}$ is the zero vector (all of its elements equal to zero). Because of linearity, the inner product between $\phi_{i}$ and each term on the right-hand side of (31) can be considered separately; thus, for the first term, the result is

$$
\begin{aligned}
\left\langle\Phi^{\top}, M \Phi \boldsymbol{D}_{\phi} \hat{\boldsymbol{x}}_{2}\right\rangle & =M \int_{0}^{T} \Phi^{\top}(t) \Phi(t) d t \boldsymbol{D}_{\phi} \hat{\boldsymbol{x}}_{2} \\
& =M \Gamma \boldsymbol{D}_{\phi} \hat{\boldsymbol{x}}_{2}=\frac{T}{2} M \boldsymbol{D}_{\phi} \hat{\boldsymbol{x}}_{2}
\end{aligned}
$$

where the matrix $\Gamma$ is defined in (27). Similarly, the remaining terms of the residual in (31) become

$$
\begin{aligned}
\left\langle\Phi^{\top}, B \Phi \hat{\boldsymbol{x}}_{2}\right\rangle & =\frac{T}{2} B I_{N} \hat{\boldsymbol{x}}_{2} \\
\left\langle\Phi^{\top}, S \Phi \hat{\boldsymbol{x}}_{1}\right\rangle & =\frac{T}{2} S I_{N} \hat{\boldsymbol{x}}_{1} \\
\left\langle\Phi^{\top}, \Phi \hat{\boldsymbol{u}}\right\rangle & =\frac{T}{2} I_{N} \hat{\boldsymbol{u}} \\
\left\langle\Phi^{\top}, f_{e}\right\rangle & =\frac{T}{2} I_{N} \hat{\boldsymbol{e}}
\end{aligned}
$$

where $\hat{e}$ is the vector of Fourier coefficients of the excitation force. The product corresponding to the convolution term in (31) requires some manipulation in order to be expressed in matrix form. Because of the linearity of the convolution integral, and of the orthogonality of the basis, the reduction can be carried out one frequency at the time; thus, it is convenient to consider $\Phi$ and $\hat{x}$ as composed of two elements only, such as

$$
\Phi^{k}=\left[\cos \left(k \omega_{0} t\right), \sin \left(k \omega_{0} t\right)\right], \quad \hat{\boldsymbol{x}}_{2}^{k}=\left[x_{2 k}^{c}, x_{2 k}^{s}\right]^{\top} .
$$

In this case, by the commutativity property of the convolution and by the fact that the impulse response $K(t)=0$ for $t<0^{2}$, the result is

$$
\int_{-\infty}^{t} K(t-\tau) \Phi^{k}(t) \hat{\boldsymbol{x}}_{2}^{k} d \tau=\int_{0}^{+\infty} K(\tau) \Phi^{k}(t-\tau) \hat{\boldsymbol{x}}_{2}^{k} d \tau
$$

By developing the product terms

$$
\begin{aligned}
\int_{0}^{+\infty} & K(\tau) \Phi^{k}(t-\tau) \hat{\boldsymbol{x}}_{2}^{k} d \tau \\
= & \int_{0}^{+\infty} K(\tau)\left(x_{2 k}^{c} \cos \left(k \omega_{0}(t-\tau)\right)\right. \\
& \left.+x_{2 k}^{s} \sin \left(k \omega_{0}(t-\tau)\right)\right) d \tau \\
= & x_{2 k}^{s} \sin \left(k \omega_{0} t\right) \int_{0}^{+\infty} K(\tau) \cos \left(k \omega_{0}(\tau)\right) d \tau \\
& -x_{2 k}^{s} \cos \left(k \omega_{0} t\right) \int_{0}^{+\infty} K(\tau) \sin \left(k \omega_{0}(\tau)\right) d \tau \\
& +x_{2 k}^{c} \cos \left(k \omega_{0} t\right) \int_{0}^{+\infty} K(\tau) \cos \left(k \omega_{0}(\tau)\right) d \tau \\
& +x_{2 k}^{c} \sin \left(k \omega_{0} t\right) \int_{0}^{+\infty} K(\tau) \sin \left(k \omega_{0}(\tau)\right) d \tau .
\end{aligned}
$$

${ }^{2}$ which corresponds to $K(t-\tau)=0$ is zero for $\tau>t$.
By applying Ogilvie's relations [13]

$$
\begin{aligned}
-\omega\left(m(\omega)-m_{\infty}\right) & =\int_{0}^{\infty} K(t) \sin (\omega t) d t \\
R(\omega) & =\int_{0}^{\infty} K(t) \cos (\omega t) d t \\
m_{\infty} & =\lim _{\omega \rightarrow \infty} m(\omega)
\end{aligned}
$$

where $m(\omega)$ and $R(\omega)$ are known as added mass and radiation resistance [14], respectively, the convolution integral becomes

$$
\begin{aligned}
\int_{0}^{+\infty} & K(\tau) \Phi^{k}(t-\tau) \hat{\boldsymbol{x}}_{2}^{k} d \tau \\
= & x_{2 k}^{s}\left(\sin \left(k \omega_{0} t\right) R\left(k \omega_{0}\right)-\cos \left(k \omega_{0} t\right)\right. \\
& \left.\times\left(-k \omega_{0}\left(m\left(k \omega_{0}\right)-m_{\infty}\right)\right)\right) \\
& +x_{2 k}^{c}\left(\cos \left(k \omega_{0} t\right) R\left(k \omega_{0}\right)+\sin \left(k \omega_{0} t\right)\right. \\
& \left.\times\left(-k \omega_{0}\left(m\left(k \omega_{0}\right)-m_{\infty}\right)\right)\right) \\
= & \Phi^{k}\left[\begin{array}{cc}
R\left(k \omega_{0}\right) & k \omega_{0} m\left(k \omega_{0}\right) \\
-k \omega_{0} m\left(k \omega_{0}\right) & R\left(k \omega_{0}\right)
\end{array}\right] \hat{\boldsymbol{x}}_{2}^{k}-m_{\infty} \Phi^{k} \boldsymbol{D}_{\phi}^{k} \hat{\boldsymbol{x}}_{2}^{k} .
\end{aligned}
$$

Before combining all the terms, it is convenient to carry out an additional substitution: by noting that the matrix $D_{\phi}$ is invertible, and its inverse is still block diagonal with blocks

$$
\boldsymbol{D}_{\phi}^{k^{-1}}=\left[\begin{array}{cc}
0 & \frac{1}{k \omega_{0}} \\
-\frac{1}{k \omega_{0}} & 0
\end{array}\right]
$$

and using (30), the variable $\hat{\boldsymbol{x}}_{1}$ in the residual (31) can be substituted with

$$
\hat{\boldsymbol{x}}_{1}=\boldsymbol{D}_{\phi}^{-1} \hat{\boldsymbol{x}}_{2} .
$$

Thus, the inner product relating to the restoring force term becomes

$$
\left\langle S \Phi \boldsymbol{D}_{\phi}^{-1} \hat{\boldsymbol{x}}_{2}, \phi_{i}\right\rangle \Rightarrow \frac{T}{2} S I_{N} \boldsymbol{D}_{\phi}^{-1} \hat{\boldsymbol{x}}_{2} .
$$

Combining all the terms of the inner product in (33), the discretized equation of motion becomes the linear system

$$
\boldsymbol{G} \hat{\boldsymbol{x}}_{2}=\hat{\boldsymbol{u}}+\hat{\boldsymbol{e}}
$$

where the matrix $G$ is block diagonal, with $2 \times 2$ blocks as

$$
\boldsymbol{G}=\left[\begin{array}{cccccc}
D_{1} & M_{1} & 0 & \cdots & 0 & 0 \\
-M_{1} & D_{1} & 0 & \cdots & 0 & 0 \\
0 & 0 & \ddots & & \vdots & \vdots \\
\vdots & \vdots & & \ddots & 0 & 0 \\
0 & 0 & \cdots & 0 & D_{N / 2} & M_{N / 2} \\
0 & 0 & \cdots & 0 & -M_{N / 2} & D_{N / 2}
\end{array}\right]
$$

and where

$$
\begin{aligned}
D_{k} & =R\left(k \omega_{0}\right)+B \\
M_{k} & =n \omega_{0}\left(m_{b}+m\left(k \omega_{0}\right)\right)-S /\left(k \omega_{0}\right)
\end{aligned}
$$

for $k=1 \ldots N / 2$. The matrix $G$ is nonsingular, since $B>0$, thus

$$
\operatorname{det} \boldsymbol{G}=\prod_{n=1}^{N / 2} D_{n}^{2}+M_{n}^{2}>0 .
$$


The equation of motion (36) can then be solved with respect to $\hat{\boldsymbol{x}}_{2}$ as

$$
\hat{\boldsymbol{x}}_{2}=\boldsymbol{G}^{-1} \hat{\boldsymbol{u}}+\boldsymbol{G}^{-1} \hat{\boldsymbol{e}}
$$

and, by substituting $\hat{\boldsymbol{x}}_{2}$ into the approximated absorbed energy $J^{N}$ in (26), we get

$$
J^{N}=-\frac{T}{2} \hat{\boldsymbol{u}}^{\top} \boldsymbol{G}^{-1} \hat{\boldsymbol{u}}-\frac{T}{2} \hat{\boldsymbol{u}}^{\top} \boldsymbol{G}^{-1} \hat{\boldsymbol{e}}
$$

which is a quadratic function of the variable $\hat{\boldsymbol{u}}$ solely. In essence, the state variables have been eliminated by substitution, and the optimization is carried out over the control variable $\hat{\boldsymbol{u}}$ only. More importantly, the constrained optimization problem given by the cost function describing the total absorbed energy $J^{N}$ in (26) and the linear equality constraints describing the system dynamics in (36) has been transformed into an unconstrained quadratic program.

Additionally, since radiation is a dissipative process [15], the radiation resistance is positive, i.e., is $R(\omega)>0$, and all the diagonal elements of the matrix $G$ are positive; thus, the symmetric part of $\boldsymbol{G},\left(\boldsymbol{G}+\boldsymbol{G}^{\boldsymbol{\top}}\right) / 2$ is positive definite and the absorbed energy function (39) is concave. Therefore, the optimal solution $\hat{\boldsymbol{u}}^{*}$ for the unconstrained problem, which minimizes (26) is then

$$
\hat{\boldsymbol{u}}^{*}=\left(\boldsymbol{G}^{-1}+\boldsymbol{G}^{-\mathrm{T}}\right)^{-1} \boldsymbol{G}^{-1} \hat{\boldsymbol{e}} .
$$

\section{A. Force and Oscillation Amplitude Constraints}

Constraints on the PTO force and the oscillation amplitude reflect physical limitations on the device or its components. In this section, we consider inequality constraints describing the maximum allowed force and maximum allowed oscillation amplitude, described as

$$
\begin{gathered}
\left|f_{\text {pto }}(t)\right| \leq F_{\max } \\
|z(t)| \leq Z_{\max }
\end{gathered}
$$

which correspond, for the approximated problem, to

$$
\begin{aligned}
|\Phi(t) \hat{\boldsymbol{u}}| & \leq F_{\max } \\
\left|\Phi(t) \hat{\boldsymbol{x}}_{1}\right| & \leq Z_{\max } .
\end{aligned}
$$

It is difficult to find the extrema of a Fourier series; thus, one possible approach to deal with the constraints in (43) and (44) is to consider their two-norm approximation, as described in [6]. An alternative approach is to enforce the constraint only at a set of specified time instants $\left\{t_{k}\right\}_{k=0}^{N_{c}}$, i.e.,

$$
\begin{aligned}
\Phi\left(t_{k}\right) \hat{\boldsymbol{u}} \leq F_{\max }, & -\Phi\left(t_{k}\right) \hat{\boldsymbol{u}} \leq F_{\max } \\
\Phi\left(t_{k}\right) \hat{\boldsymbol{x}}_{1} \leq Z_{\max }, & -\Phi\left(t_{k}\right) \hat{\boldsymbol{x}}_{1} \leq Z_{\max } .
\end{aligned}
$$

for $k=0, \ldots, N_{c}$. By defining the vector $\Theta$ as

$$
\boldsymbol{\Theta}=\left[\begin{array}{c}
\Phi_{0} \\
\Phi_{1} \\
\vdots \\
\Phi_{N_{c}}
\end{array}\right]
$$

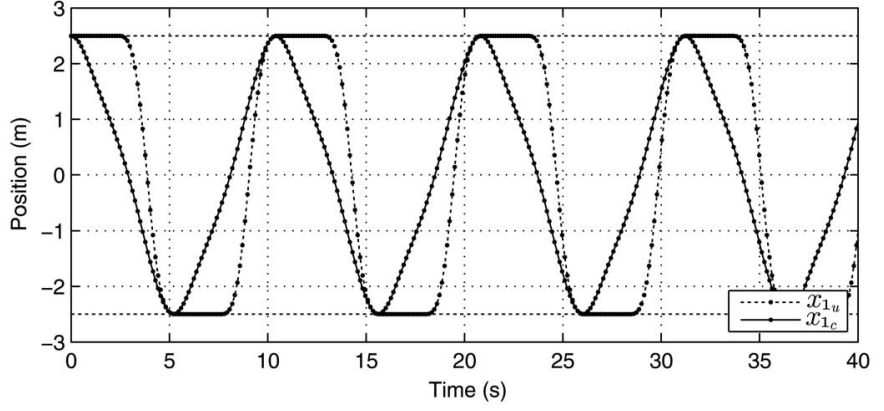

Fig. 3. Vertical position for an incident regular wave of period $T_{w}=10 \mathrm{~s}$ and amplitude $A=5 \mathrm{~m}: x_{1_{u}}$ is the vertical position when the PTO force is not constrained; $x_{1_{c}}$ is the vertical position when the PTO force is constrained.

the constraints in (45) and (46) can be approximated by the linear inequalities

$$
\begin{aligned}
& {\left[\begin{array}{c}
\boldsymbol{\Theta} \\
-\boldsymbol{\Theta}
\end{array}\right] \hat{\boldsymbol{u}} \leq \mathbf{1}_{2\left(N_{c}+1\right) \times 1} F_{\max }} \\
& {\left[\begin{array}{c}
\boldsymbol{\Theta} \\
-\boldsymbol{\Theta}
\end{array}\right] \hat{\boldsymbol{x}}_{1} \leq \mathbf{1}_{2\left(N_{c}+1\right) \times 1} Z_{\max }}
\end{aligned}
$$

where $\mathbf{1}_{2\left(N_{c}+1\right) \times 1}$ is the vector of all ones of size $2\left(N_{c}+1\right)$. The inequality constraint, relative to the position in (46), can be expressed as a function of $\hat{\boldsymbol{u}}$ using (35) and (38), as

$$
\left[\begin{array}{c}
\boldsymbol{\Theta} \\
-\boldsymbol{\Theta}
\end{array}\right] D_{\phi}^{-1} \boldsymbol{G}^{-1} \hat{\boldsymbol{u}} \leq \mathbf{1}_{2\left(N_{k} \times 1\right)} Z_{\max }-\left[\begin{array}{c}
\boldsymbol{\Theta} \\
-\boldsymbol{\Theta}
\end{array}\right] \boldsymbol{D}_{\phi}^{-1} \boldsymbol{G}^{-1} \hat{\boldsymbol{e}}
$$

In summary, the resulting constrained optimal control problem is the convex quadratic program defined by the cost function $J^{N}$ in (39), subject to the linear inequality constraints on the PTO force in (47) and oscillation amplitude in (49).

\section{B. Simulation Results}

This section presents simulation results for the heavingbody point absorber WEC depicted in Fig. 1, specified as a vertical cylinder of radius $r=4 \mathrm{~m}$ and draught $d=10 \mathrm{~m}$. Both regular and irregular incident waves are considered, and PTO force and oscillation amplitude constraints are respected. Figs. 3-5 depict results for a monochromatic incident wave with period $T_{r}=10 \mathrm{~s}$ and amplitude $A=5 \mathrm{~m}$. The expansions for the approximation of the state in (24) and the control in (25) are composed of 40 frequency components, corresponding to $N=80$. The oscillation amplitude constraint has been set to $Z_{\max }=2.5 \mathrm{~m}$, while several values of the PTO force constraints have been considered, that is $F_{\max }=\{1,1.25,1.5,2\} \cdot 10^{6} \mathrm{~N}$. The time instants $t_{k}$ have been chosen equally spaced, with a distance 10 times smaller than the smallest period of the Fourier expansion, i.e., $t_{k+1}-t_{k}=2 T / 10 N$. Thus, for the simulation considered in this section, $N c=1+T /\left(t_{k+1}-t_{k}\right)=5 N+1=401$.

Each plot in Figs. 3-5 depicts two sets of curves: the curves associated with the variables with subscript " $c$ " (i.e., $x_{1_{c}}, x_{2_{c}}, u_{c}$ ) denote the simulation results when the PTO force is constrained with the most stringent value $\left(F_{\max }=\right.$ $1 \times 10^{6} \mathrm{~N}$ ), whereas the curves associated with the variables 


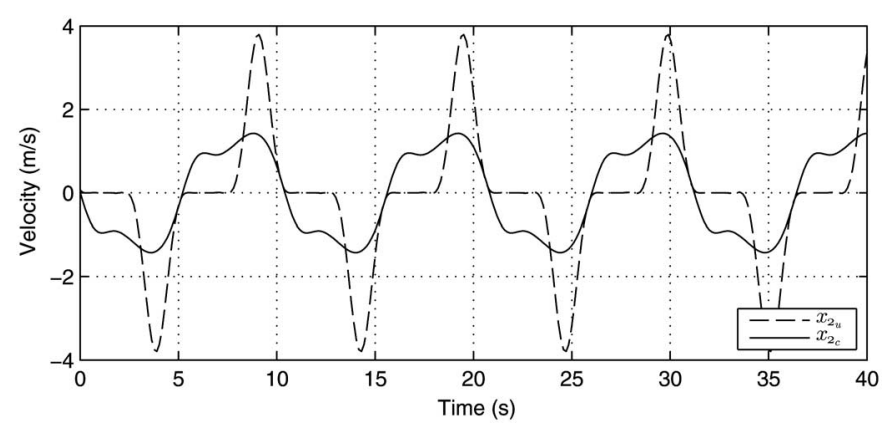

Fig. 4. Vertical velocity for an incident regular wave of period $T_{w}=10 \mathrm{~s}$ and amplitude $A=5 \mathrm{~m}: x_{2}$ is the vertical velocity when the PTO force is not constrained; $x_{2_{c}}$ is the vertical velocity when the PTO force is constrained.

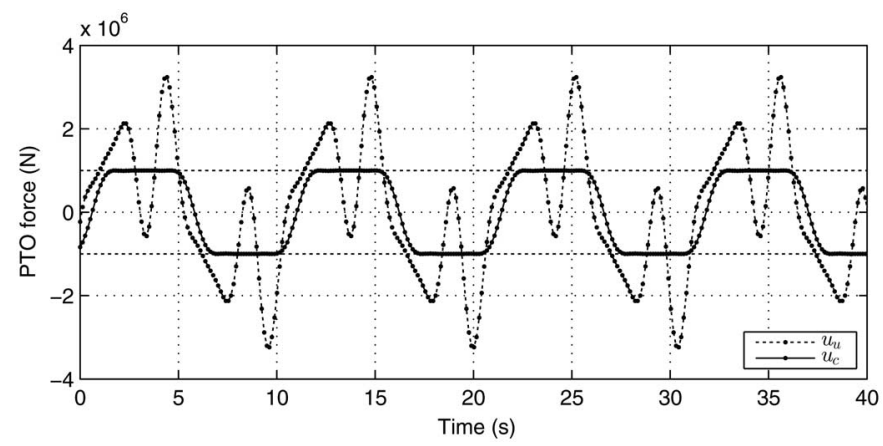

Fig. 5. Unconstrained PTO force $u_{u}$ and constrained PTO force $u_{c}$ for an incident regular wave of period $T_{w}=10 \mathrm{~s}$ and amplitude $A=5 \mathrm{~m}$.

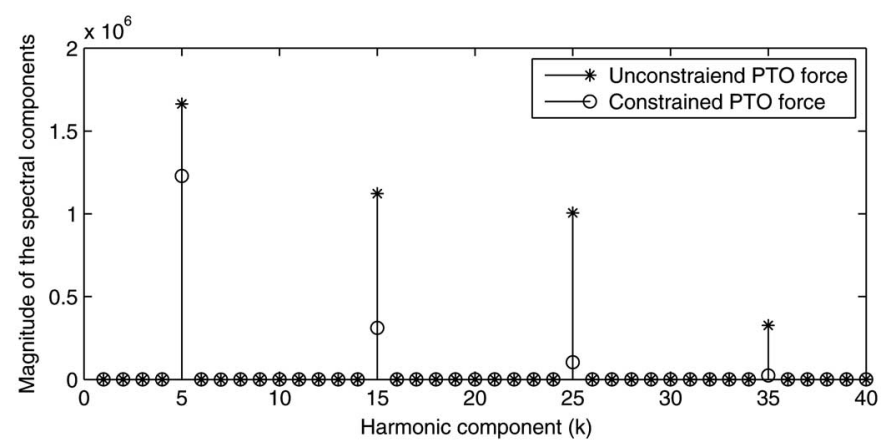

Fig. 6. Spectral components of the PTO force.

with subscript " $u$ " (i.e., $x_{1_{u}}, x_{2_{u}}, u_{u}$ ) denote the simulation results when the PTO force is unconstrained. The "dot" marks superimposed on the plots of the positions $\left(x_{1_{c}}, x_{1_{u}}\right)$ in Fig. 3 and the PTO forces in Fig. 5 denote the time points $t_{k}$ at which the constraints are being enforced, via (47) and (49), for the PTO force and device position, respectively.

It is interesting to note that both the optimal motion of the device and the optimal time profile of the PTO force are smoother when the PTO force is strongly constrained, thus, the device is subject to less mechanical stress. Fig. 6 shows the frequency components of the optimal PTO force for the situation where the PTO force is unconstrained $(*$ mark) and when the most stringent constraint is active ( $\circ$ mark), confirming the previous observation. In fact, the unconstrained PTO force is characterized by large values of higher frequency components up to the seventh harmonic, whereas the harmonic content of the constrained PTO force is considerably less.

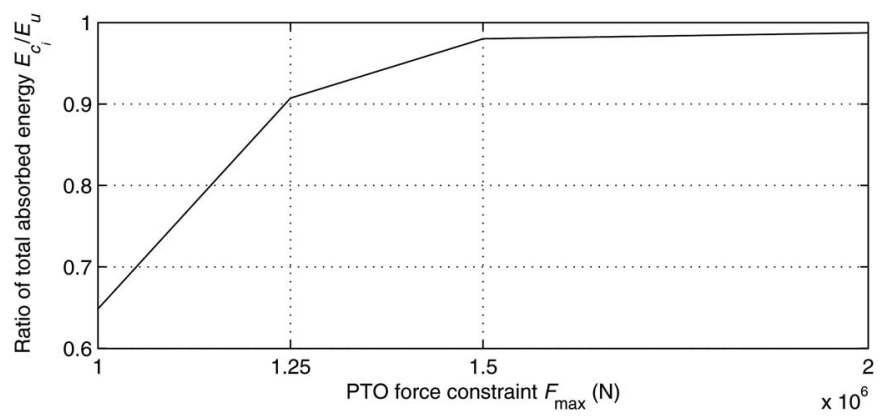

Fig. 7. Total absorbed energy as a function of the force constraint.

Fig. 6 also shows that the optimal profiles of both the constrained and the unconstrained PTO forces only contain odd harmonics, when the position constraints are active. For the real-time implementation, it could be beneficial to reduce the dimension of the NLP problem by eliminating all the frequencies for which the energy is zero or negligible. Due to the orthogonality of the Fourier series, this could be achieved by looking at the vector of Fourier coefficients of the excitation force $(\hat{e})$, and by solving the optimization problem considering only the frequencies which contain "most" of the energy and their harmonics. The reduced problem will consist of the quadratic program with the cost function

$$
J^{N}=-\frac{T}{2} \tilde{\boldsymbol{u}}^{T} \widetilde{\boldsymbol{G}}^{-1} \tilde{\boldsymbol{u}}-\frac{T}{2} \tilde{\boldsymbol{u}}^{T} \widetilde{\boldsymbol{G}}^{-1} \tilde{\boldsymbol{e}}
$$

in place of (39), where $\tilde{\boldsymbol{u}}$ and $\tilde{\boldsymbol{e}}$ are the "reduced" vectors of Fourier coefficients of the control signal and excitation force, respectively, which are built by extracting the elements of $\hat{\boldsymbol{u}}$ and $\hat{e}$ for which the energy is nonzero, and their harmonics.

The matrix $\widetilde{G}$ is derived from the matrix $G$ in a similar manner by extracting the rows and columns corresponding to the frequencies, which contain most of the energy, and their harmonics.

Fig. 7 depicts the ratio $E_{c} / E_{u}$ of the total absorbed energy for several values of the PTO force constraints $E_{c}$ and the energy absorbed in the unconstrained case $E_{u}$. With a force constraint of $1.25 \times 10^{6} \mathrm{~N}$, which is $30 \%$ of the maximum value of the PTO force in the unconstrained case (see Fig. 5), it is still possible to absorb around $90 \%$ of the energy that is absorbed when the PTO force is not constrained. This analysis plays an important role in the techno-economical optimization of WECs, since absorbed energy is a major determinant of the revenue generated by the device and the power rating is strongly related to the cost of the device.

Simulation results for irregular waves, corresponding to a Bretschneider spectrum of peak period $T_{p}=10 \mathrm{~s}$ and significant wave height $H_{s}=1 \mathrm{~m}$, are depicted in Figs. 8-10. In this case, the approximations of the state and control in (24) and (25) are composed of 80 frequencies $(N=160)$, the oscillation amplitude has been restricted to $Z_{\max }=2.5 \mathrm{~m}$ and the PTO force to $F_{\max }=5.3 \times 10^{5} \mathrm{~N}$.

With regard to the effect of constraints on the motion of the device (Figs. 3 and 4) and PTO force, the observations described for the regular incident wave still apply; namely, that a more stringent PTO force constraint results in 


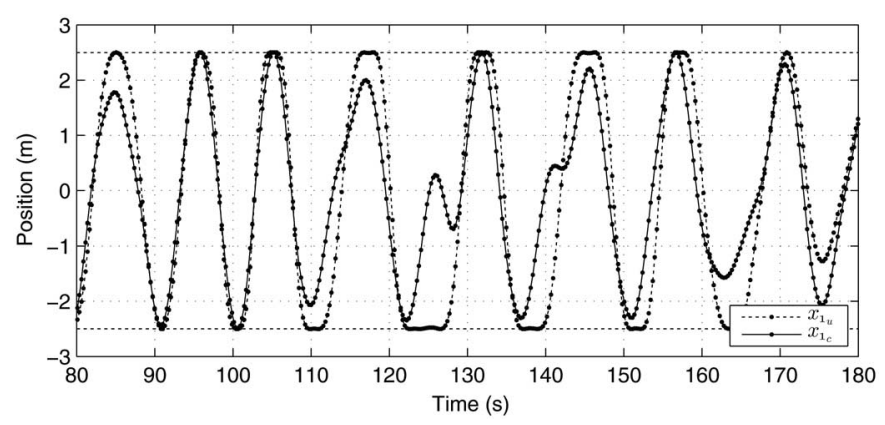

Fig. 8. Vertical position for a Bretschneider sea state of $T_{p}=10 \mathrm{~s}$ and $H_{s}=$ $1 \mathrm{~m}: x_{1_{u}}$ is the vertical position when the PTO force is not constrained; $x_{1_{c}}$ is the vertical position when the PTO force is constrained.

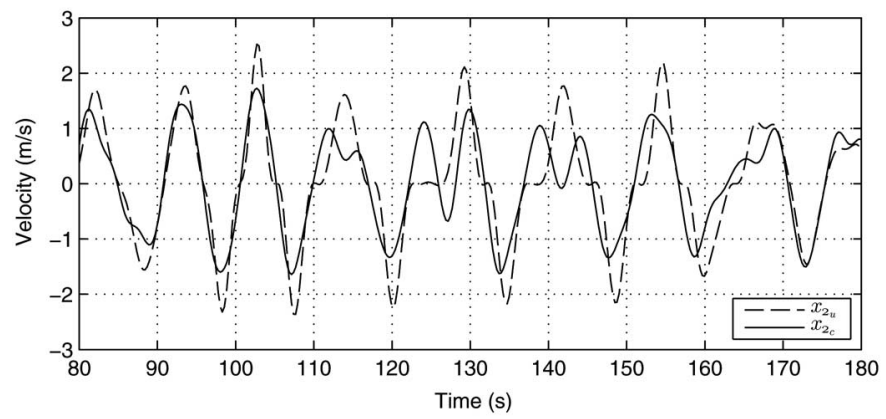

Fig. 9. Vertical velocity for a Bretschneider sea state of $T_{p}=10 \mathrm{~s}$ and $H_{s}=$ $1 \mathrm{~m}: x_{2_{u}}$ is the vertical velocity when the PTO force is not constrained; $x_{2_{c}}$ is the vertical velocity when the PTO force is constrained.

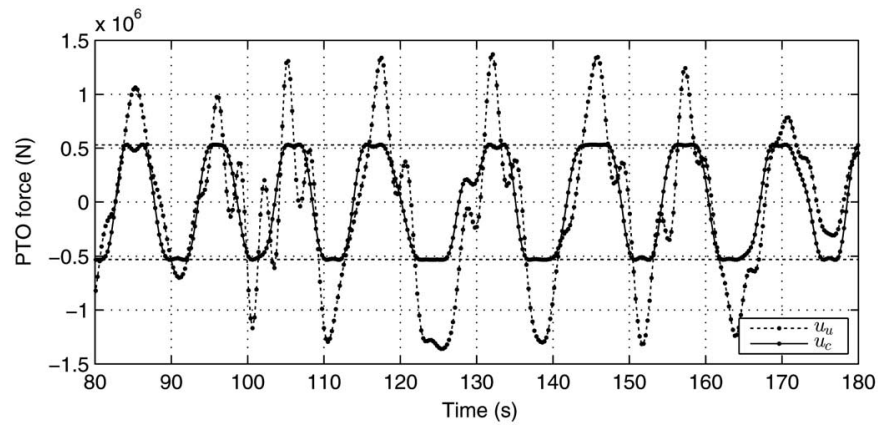

Fig. 10. Unconstrained PTO force $u_{u}$ and constrained PTO force $u_{c}$ for a Bretschneider sea state of $T_{p}=10 \mathrm{~s}$ and $H_{s}=1 \mathrm{~m}$.

a smoother motion and PTO force profile. Additionally, setting the PTO force constraint to $50 \%$ of the maximum unconstrained PTO force reduces the total absorbed energy by only $10 \%$, approximately.

\section{Computational CONSIDERATions}

In the unconstrained case, the optimal solution to the energy maximization problem is a straightforward algebraic calculation, as shown in (40). The dimension of this matrix calculation is a function of the number of bodies, the number of degrees of freedom for each body, and the number of frequencies $N / 2$ used in the discretization approximation of the system variables.

In the constrained case, an NLP problem results with the performance function in (39), subject to the linear inequality constraints on the PTO force in (47) and oscillation amplitude in (49). In addition to the WEC system dimension and frequencies associated with the unconstrained optimization problem, the computational load for the constrained case additionally depends on $N_{c}$, the number of time instants at which the constraints are imposed.

In order to achieve a parsimonious computational problem, $N$ and $N_{c}$ must be chosen judiciously. Application-specific experience is required to choose a minimum value of $N_{c}$ so that constraints are not compromised, while there is a considerable scope in pruning $N$ by inspection, as evidenced by Fig. 6 . In particular, the value of $N$ should be selected in combination with the fundamental frequency $\omega_{0}$ of the Fourier expansion ${ }^{3}$; the values of $\omega_{0}$ and $N$ can be selected, e.g., by looking at the power spectrum of the wave elevation (or the excitation force). The frequency $\omega_{0}$ should be smaller than the lowest frequency with significant energy content and, conversely, $N$ should be chosen such that $N \omega_{0} / 2$ is larger than the larger frequency with significant energy content. Moreover, when force and amplitude constraints become active, they introduce higher harmonics in the motion and force (see e.g., Fig. 6), therefore, the choice of $N$ should also take this aspect into consideration. In practice, the smaller the value of $\omega_{0}$, the greater is the frequency resolution, as the Fourier components are at integer multiples of the fundamental frequencies; however, a higher frequency resolution requires a larger value of $N$, which corresponds to a larger dimension of the NLP problem.

A significant computational saving, however, is always achievable by reconfiguring the radiation damping convolution calculation into an offline and online components, as shown in the next section.

\section{A. Radiation Convolution Integral}

In Section III, it is shown that the convolution integral describing the radiation force can be solved analytically when the state (velocity) is approximated using a truncated Fourier series; thus, the numerical integration of the convolution integral can be avoided when solving the equation of motion during the solution of the NLP problem. It should be noted that whenever the velocity is approximated by a linear combination of basis functions, the numerical integration of the convolution integral can be done offline, for a given hydrodynamic problem and a given choice of basis functions. In fact, considering the expansion for the velocity in (9), i.e.,

$$
v_{i}(t) \approx v_{i}^{N}(t)=\sum_{k=1}^{N} x_{i k}^{v} \phi_{k}(t), \quad i=1, \ldots, n
$$

the term of the residual of the dynamic equation involving the convolution integral in (16) is

$$
\begin{aligned}
& \sum_{j=1}^{n} \int_{0}^{t} K_{i j}(t-\tau) v_{j}^{N}(\tau) d \tau \\
& \quad=\sum_{j=1}^{n} \sum_{k=1}^{N} x_{i k}^{v} \int_{0}^{t} K_{i j}(t-\tau) \phi_{k}(\tau) d \tau .
\end{aligned}
$$

\footnotetext{
${ }^{3}$ is related to the period $T$ over which the energy is being optimized as $\omega_{0}=2 \pi / T$.
} 
The minimization of the residual by means of the meanweighted residual method results in the inner products $\left\langle r_{i}^{v}, \psi_{j}\right\rangle$ in (18); because of linearity, i.e., $\langle f+g, h\rangle=\langle f, h\rangle+\langle g, h\rangle$, each of the inner products corresponding to the convolution integral can be considered separately, and they are

$$
\begin{aligned}
& \left\langle\int_{0}^{t} K_{i j}(t-\tau) \phi_{k}(\tau) d \tau, \psi_{l}(t)\right. \\
& \quad=\int_{0}^{T} \int_{0}^{t} K_{i j}(t-\tau) \phi_{k}(\tau) d \tau \psi_{l}(t) d t=\kappa_{i j k l} .
\end{aligned}
$$

The elements $\kappa_{i j k l}$ are not functions of time; they only depends on the basis functions $\phi_{k}$, the test functions $\psi_{k}$, and the radiation impulse responses $K_{i j}$. Consequently, the terms of the inner products $\left\langle r_{i}^{v}, \psi_{j}\right\rangle$ in (18) can be written as

$$
\left\langle\sum_{j=1}^{n} \int_{0}^{t} K_{i j}(t-\tau) v_{j}^{N}(\tau) d \tau, \psi_{l}(t)\right\rangle=\sum_{j=1}^{n} \sum_{k=1}^{N} x_{i k}^{v} \kappa_{i j k l} .
$$

The coefficients $\kappa_{i j k l}$ can be calculated either analytically, if possible, or by numerical integration once the basis functions, test functions, and the hydrodynamics have been chosen. In other words, by applying the mean-weighted residual method for the approximation of the equation of motion, all the computation involving the integration of the convolution integral can be carried out offline, reducing the computational load when solving the NLP problem of the approximated optimal control problem

\section{CONCLUSION}

This paper presents a general mathematical framework for the solution of the WEC control problem. The flexibility of the approach permits a wide variety of basis functions for variable (motion and PTO force) parameterization to be employed, while the degree of approximation (choice of $N$ ) provides a tradeoff between approximation accuracy and computational complexity. Such complexity scaling makes the algorithm ideally suited to real-time control and this paper shows how inspection of the PTO spectrum can result in considerable computational savings with minimal reduction in approximation fidelity. Other key features include the efficient representation of the radiation damping convolution, which further improves the computational attractiveness of the method. While the simulation results included are for the single-body case, multibody WEC systems and WEC arrays are easily accommodated using an appropriate model and PTO configuration matrix, as outlined in Section II-A, with simulation examples for the two-body case shown in [10] and an application to the control of WEC arrays shown in [16].

\section{REFERENCES}

[1] H. Eidsmoen, "Optimum control of a floating wave-energy converter with restricted amplitude," J. Offshore Mech. Arct. Eng., vol. 118, no. 2, pp. 96-102, 1996.
[2] J. Hals, J. Falnes, and T. Moan, "Constrained optimal control of a heaving buoy wave-energy converter," J. Offshore Mech. Arct. Eng., vol. 133, no. 1 , p. 011401,2011

[3] J. A. M. Cretel, G. Lightbody, G. P. Thomas, and A. W. Lewis, "Maximisation of energy capture by a wave-energy point absorber using model predictive control," in Proc. 18th IFAC World Congr., 2011, pp. 3714-3721.

[4] G. Li and M. Belmont, "Model predictive control of sea wave energy converters-Part I: A convex approach for the case of single device," Renew. Energy, vol. 69, pp. 453-463, 2014.

[5] B. A. Finlayson and L. E. Scriven, "The method of weighted residualsreview," Appl. Mech. Rev., vol. 19, no. 9, pp. 735-748, Sep. 1966.

[6] G. Bacelli, J. V. Ringwood, and J.-C. Gilloteaux, "A control system for a self-reacting point absorber wave energy converter subject to constraints," in Proc. IFAC World Congr. Milan, vol. 18, 2011, pp. 11387-11392.

[7] D. R. Herber and J. T. Allison, "Wave energy extraction maximization in irregular ocean waves using pseudospectral methods," in Proc. 2013 ASME Des. Eng. Tech. Conf., No. DETC2013-12600, ASME, Portland, OR, USA, 2013.

[8] W. Cummins, "The impulse response function and ship motions," Schiffstechnik, vol. 9, pp. 101-109, 1962.

[9] G. Elnagar, M. Kazemi, and M. Razzaghi, "The pseudospectral legendre method for discretizing optimal control problems," IEEE Trans. Autom. Control, vol. 40, pp. 1793-1796, 1995.

[10] G. Bacelli, "Optimal control of wave energy converters," Ph.D. dissertation, Dept. Electronic Engineering, National Univ. Ireland, Maynooth, Ireland, 2014

[11] G. Bacelli and J. Ringwood, "Nonlinear optimal wave energy converter control with application to a flap-type device," in Proc. 19th IFAC World Congr., 2014, pp. 7696-7701.

[12] K. Budal and J. Falnes, "A resonant point absorber of ocean-wave power," Nature, vol. 256, no. 5517, pp. 478-479, Aug. 1975 (with corrigendum in vol. 257, p. 626).

[13] T. Ogilvie, "Recent progress toward the understanding and prediction of ship motions," in Proc. 5th Symp. Nav. Hydrodyn., Bergen, Norway, 1964, pp. 3-79.

[14] J. Falnes, Ocean Waves and Oscillating Systems: Linear Interactions Including Wave-Energy Extraction. Cambridge, U.K.: Cambridge Univ. Press, 2002.

[15] C. J. Damaren, "Time-domain floating body dynamics by rational approximation of the radiation impedance and diffraction mapping," Ocean Eng., vol. 27, no. 6, pp. 687-705, Jun. 2000.

[16] G. Bacelli, P. Balitsky, and J. V. Ringwood, "Coordinated control of arrays of wave energy devices-Benefits over independent control," IEEE Trans. Sustain. Energy, vol. 4, no. 4, pp. 1091-1099, Oct. 2013.

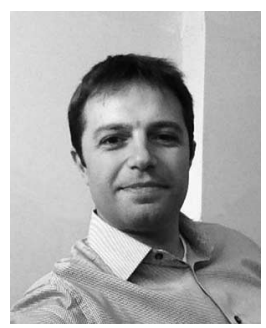

Giorgio Bacelli received the Laurea Magistrale in electronic engineering from the Universit Politecnica delle Marche, Ancona, Italy, in 2006, and the Ph.D. degree in electronic engineering from the Centre for Ocean Energy Research, Maynooth University, Maynooth, Ireland, in 2014.

Currently, he is with Sandia National Laboratories, Albuquerque, NM, USA, working on the design and implementation of control strategies for wave energy converters.

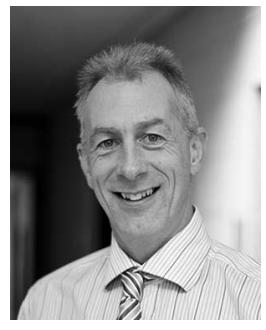

John V. Ringwood (M'87-SM'97) received the Diploma in electrical engineering from Dublin Institute of Technology, Dublin, Ireland, and the $\mathrm{Ph} . \mathrm{D}$. degree in control systems from Strathclyde University, Glascow, U.K.

Previously, he was with the School of Electronic Engineering, Dublin City University, Dublin, Ireland, and has held visiting positions with Massey University, New Zealand, and Auckland University, Auckland, New Zealand. He is the Professor of Electronic Engineering and Director with the Centre for Ocean Energy Research, Maynooth University, Maynooth, Ireland. Dr. Ringwood is a Chartered Engineer and a Fellow of Engineers Ireland. 